\title{
Prevalência de infecção por enteroparasitoses e caracterização socioeconômica de colônias de pescadores brasileiros
}

\author{
Prevalence of intestinal parasitic infection and socioeconomic characterization of Brazilian fishing \\ colonies
}

Prevalencia de parasitosis intestinal y caracterización socioeconómica de las colonias pesqueras brasileñas

\section{Resumo}

Introdução: a pesca artesanal é uma atividade que pode trazer prejuízos à saúde do trabalhador, pois expõe o pescador a diversas intempéries e elevada carga de esforço físico, deixando-os susceptíveis a doenças infeciosas, como as parasitoses intestinais. Objetivo: identificar a maior prevalência de infecção por enteroparasitoses em pescadores da região norte do Brasil. Métodos: estudo transversal descritivo, de acordo com as diretrizes da Declaração STROBE, realizado em duas colônias de pescadores na região Norte do Brasil. Resultados: identificamos os seguintes protozoários: Escherichia Coli, Endolimax Nana, Escherichia Coli, Giardia Lamblia, Iodamoeba Bustschlii. Não foram encontradas amostras positivas para helmintoses. Conclusão: entre as espécies de enteroparasitoses investigadas no ano de 2019, na região Norte do Brasil, houve prevalência (> 50\%) de Endolimax Nana em pescadores parasitados. Palavras-chave: Doenças parasitárias; Prevalência; Populações vulneráveis.

\begin{abstract}
Overview: artisanal fishing is an activity that can harm workers' health, as it exposes fishermen to various weather conditions and high physical effort, leaving them susceptible to infectious diseases, such as intestinal parasites. Objective: identify the highest prevalence of infection by intestinal parasites in fishermen in the northern region of Brazil. Methods: descriptive cross-sectional study, in accordance with the guidelines of the STROBE Declaration, carried out in two fishing colonies in Northern Brazil. Results: we identified the following protozoa: Escherichia Coli, Endolimax Nana, Escherichia Coli, Giardia Lamblia, Iodamoeba Bustschlii. No positive samples for helmintoses were found. Conclusion: among the species of enteroparasitosis investigated in 2019, in the North of Brazil, there was a prevalence $(>50 \%)$ of Endolimax Nana in parasitized fishermen.
\end{abstract}

Keywords: Parasitic diseases; Prevalencia; Poblaciones vulnerables.

\section{Resumen}

Introducción: la pesca artesanal es una actividad que puede perjudicar la salud de los trabajadores, ya que expone a los pescadores a diversas condiciones climáticas y alto esfuerzo físico, dejándolos susceptibles a enfermedades infecciosas, como los parásitos intestinales. Objetivo: identificar la mayor prevalencia de infección por parásitos intestinales en pescadores de la región norte de Brasil. Métodos: estudio descriptivo transversal, de acuerdo con los 
lineamientos de la Declaración STROBE, realizado en dos colonias pesqueras en el norte de Brasil. Resultados: identificamos los siguientes protozoos: Escherichia Coli, Endolimax Nana, Escherichia Coli, Giardia Lamblia, Iodamoeba Bustschlii. No se encontraron muestras positivas para helmintoses. Conclusión: entre como espécies de enteroparasitosis investigadas no ano de 2019, na região Norte do Brasil, houve prevalência (> 50\%) de Endolimax Nana em pescadores parasitados.

Palabras clave: Enfermedades parasitarias, Prevalencia, Poblaciones vulnerables.

\section{Introdução}

A pesca é uma atividade comercial de relevância econômica e regulamentada pela Política Nacional de Desenvolvimento Sustentável da Agricultura e da Pesca - lei n ${ }^{\circ} 11.959 / 2009$. Os pescadores artesanais exercem a atividade pesqueira de forma autônoma, no Brasil, essa categoria representa 90,3\% $(n=440.266)$ dos pescadores, uma parcela destes está distribuída na bacia Tocantins/Araguaia que abriga aproximadamente $40 \%$ dos pescadores artesanais em águas interiores do Brasil (Brasil, 2019; Campos \& Chaves, 2016).

O registro geral de pescadores no Estado do Tocantins mostra 7.140 pescadores em atividade, distribuição de $67 \%$ para o sexo masculino, média de 41 anos e 73,2\% analfabetos com predomínio dos homens 69,4\%. Esta população está distribuída em 34 colônias de pescadores localizadas em seis regiões tocantinenses: Bico do Papagaio, Cantão/Araguaia, Norte Araguaia, Estreito, Central e Sul (Brasil, 2019; Sousa, 2013). Os pescadores artesanais ou de subsistência localizam-se próximos a rios e igarapés, muitos destes locais estão à margem de políticas públicas voltadas para à pesca artesanal e promoção da saúde dos pescadores, como saneamento básico, sem essa iniciativa a população fica vulnerável à incidência e prevalência de doenças infecciosas (SOUSA et al., 2017).

As infecções parasitárias intestinais representam parte das doenças infecciosas em humanos, e mais de dois milhões de pessoas estão infectadas por algum tipo de parasito - esse cenário é comum nos países em desenvolvimento e é responsável por pelo menos 2/3 da mortalidade mundial (Marzagão et al., 2010). As parasitoses intestinais são doenças infecciosas que afetam a saúde e representam a sexta causa de morbidade no Brasil, responsáveis por 776.358 internações hospitalar no ano de 2014 (Cruz et al., 2011). As enteroparasitoses são típicas de populações marginalizadas, e em virtude do precário saneamento básico, alguns parasitos de interesse médico são transmitidos principalmente pela água e alimentos contaminados (Andrade et al., 2010; Dos Santos Carvalho et al., 2002), mas também por contato sexual (anal e oral) e contato com a pele, haja vista que alguns parasitos depositam formas infectantes no solo (Andrade et al., 2010; Sousa et al., 2017).

Os agentes etiológicos das parasitoses intestinais são maciçamente os helmintos e protozoários que colonizam o aparelho digestório do hospedeiro e se nutrem dos nutrientes fornecidos pelos indivíduos infectados, comprometendo o estado nutricional do hospedeiro (Santos et al., 2017). Neste contexto, a pesca artesanal é uma atividade extenuante que pode trazer alguns prejuízos à saúde do trabalhador, pois expõe o pescador a diversas intempéries, a elevada carga de esforço físico, deixando-os susceptíveis às doenças ocupacionais e ou oportunistas e infeciosas (Freitas \& Rodrigues, 2015).

Assim, com base na hipótese de que a prevalência de infecções por enteroparasitoses se relaciona com os aspectos sociodemográficos, socioeconômicos, sanitário e educacional dos pescadores, emitimos a seguinte pergunta: qual é a prevalência de infecção por enteroparasitoses e sua relação com os fatores socioeconômicos, demográficos e sanitários em comunidades pesqueiras no Tocantins? Este estudo objetiva estimar a prevalência de infecção por enteroparasitoses e relaciona-la com as condições sanitária, socioeconômica e demográfica dos pescadores.

\section{Metodologia}

\section{Desenho do estudo}

Estudo epidemiológico, descritivo, transversal de acordo com as diretrizes da declaração STROBE realizado em duas colônias de pescadores do Tocantins no período de junho a setembro 2019. A iniciativa Strengthening the Reporting of 
Observational Studies in Epidemiology (STROBE), corresponde uma lista de recomendações fundamentais que valorizam os estudos observacionais e ou epidemiológicos a fim de contribuir para um relato mais adequado do estudo, facilitando a leitura crítica dos leitores do artigo (Malta et al., 2010).

\section{Local de estudo}

Um inquérito coproparasitológico foi realizado no mês de junho de 2019 com os pescadores das colônias de pescadores de Ipueiras e Porto Nacional no estado do Tocantins. As colônias de pescadores são comunidades localizadas às margens do rio Tocantins mantendo a atividade pesqueira de forma organizada e registros dos pescadores (Brasil, 2019; IBGE, 2010a). Vale apena que o rio que banha essas comunidades atualmente possui as características de um grande lago, essa mudança aconteceu por intervenção humana com a construção do conjunto de centrais hidrelétricas patrocinadas pela Companhia de Energia Elétrica do Estado do Tocantins, que com a construção da hidrelétrica de lajeado mudou a configuração geográfica dessa região (IBGE, 2010a).

A atividade econômica predominante nessa região é o agronegócio, todavia a pesca tem um grande fator de impacto na renda familiar dos moradores nessa região, principalmente na economia familiar subsistente, a renda per capta é de $1 / 2$ salário mínimo, o tratamento do esgoto nessa região tem média de $65 \%$ e, média de internações hospitalares por infecções paritárias intestinais de 1,2 internações por mil habitantes (Brasil, 2019; IBGE, 2010b).

\section{Seleção da amostra}

Participaram da pesquisa, pescadores artesanais moradores das cidades de Ipueiras e Porto Nacional do estado do Tocantins, cadastrados em suas respectivas colônias de pescadores, que aceitaram participar voluntariamente do estudo mediante a assinatura do Termo de Consentimento livre e Esclarecido. E, excluídos os pescadores que estavam em situação irregular no cadastro geral de pescadores em suas colônias ou estavam ausentes no momento da coleta de fezes e/ou aqueles que por motivos pessoais não assinaram o Termo de Consentimento Livre e Esclarecido.

\section{Amostra}

O total de pescadores cadastrados nas colônias de pescadores em junho de 2019 foi de 132 pescadores sendo, 77 pescadores em Ipueiras e 55 pescadores em Porto Nacional. Foram elegíveis a participar da pesquisa pelos critérios de inclusão 77 pescadores sendo, 45 pescadores de Ipueiras e 32 pescadores de Porto Nacional, o que corresponde a 58,33\% dos pescadores cadastrados.

\section{Aproximação com os Participantes da Pesquisa}

O contato com os pescadores foi mediado pelos presidentes das respectivas colônias de pescadores. Fez-se 03 reuniões em cada colônia, nestas, foram feitos os esclarecimentos sobre objetivo da pesquisa, o convite de participação mediante a assinatura do Termo de Consentimento Livre e Esclarecido (TCLE), a coleta das amostras de fezes, aplicação dos questionários e a entrega os resultados dos exames parasitológicos das fezes.

\section{Encontros}

Durante o primeiro encontro foram realizadas palestras com os participantes para expor o objetivo da pesquisa, com esclarecimentos sobre o que são parasitoses intestinais, quais os prejuízos que elas podem trazer a saúde dos pescadores, sobre os benefícios e possíveis riscos da pesquisa para os pesquisados e fizemos o convite informal para os pescadores participarem da pesquisa, foi realizado a entrega dos frascos coletores de fezes e esclarecidos as orientações sobre a coleta das fezes. No 
segundo encontro, foi feito o convite formal mediante a assinatura do Termo de Consentimento Livre e Esclarecido (TCLE), foi realizado a aplicação dos questionários e o recolhimento das amostras das fezes. No terceiro encontro reunimos para fazer a devolução dos resultados dos exames para os pescadores por intermédio da secretaria de saúde dos municípios e agradecer a participação de todos os envolvidos por intermédio dos respectivos presidentes das colônias de pescadores.

\section{Aplicação dos questionários}

Para a coleta dos dados sociodemográfico dos participantes foi utilizado o instrumento de coleta de dados do Questionário de Classificação Econômica do Brasil(Meirelles et al., 2010). Os dados socioeconômico-demográficos foram obtidos por meio da aplicação do questionário com as seguintes variáveis independentes: sexo; idade; escolaridade; condições de moradia acrescidas da classificação sugerida pela Associação Brasileira de Empresas de Pesquisa (ABEP)(Marques, 2003).

\section{Coleta das fezes}

As fezes foram coletadas a fresco, em frascos coletores universais e acondicionas em caixas térmicas para serem processadas. As análises das amostras de fezes foram realizadas por alunos do curso de Biomedicina e Enfermagem do CEULP/ULBRA no Laboratório Universitário de Análises Clínicas da própria Instituição.

\section{Equipamentos utilizados}

As fezes foram processadas para realização do exame parasitológico pelo método a fresco, utilizando as técnicas de sedimentação espontânea em água de Hoffman (1934) e centrífugo-flutuação de Faust (1939). A visualização ocorreu por microscopia óptica (100X e 400X) por leitura dupla para checagem das estruturas parasitárias. Esses métodos de diagnóstico são de baixo custo e eficientes para identificar a maioria dos enteroparasitoses (Neves et al., 2005).

\section{Desfecho}

O desfecho analisado foi à prevalência de parasitoses intestinais nos pescadores, mediante inquérito parasitológico de fezes com microscopia óptica.

\section{Métodos estatísticos}

Os dados foram tabulados por meio do software Microsoft Excel (versão 2016), em dupla digitação, e posteriormente analisados utilizando o software Stata (versão 14.2). Para verificar a normalidade dos dados quantitativos, foi utilizado o teste Shapiro Wilk e foram apresentados por médias e desvio padrão. Os dados qualitativos foram apresentados por valores absolutos e relativos. Para verificar a associação entre Parasitoses e Aspectos econômicos, demográficos e sanitários, foi utilizado o teste de Qui-quadrado, considerando a significância de $\mathrm{p}<0,05$.

\section{Princípios éticos}

A participação dos pescadores foi voluntária e deu-se após obtenção do consentimento livre e informado pelo reesposáveis. Todos os procedimentos deste estudo foram aprovados pelo comitê de ética em pesquisa com seres humanos (parecer: 3219733).

\section{Vieses}

Evidencia-se como viés a pouca adesão do público para participação da pesquisa, a dificuldade do público para o entendimento do teor relevância da pesquisa decorrente do pouco conhecimento sobre a importância das ações de promoção de 
saúde, o difícil aceso às colônias de pescadores pela distância de trafego, também a dificuldade de se reunir os pesquisados em maiores quantidades muitos deles justificados pela jornada de trabalho excessiva e praticamente em todos os dias da semana.

\section{Resultados}

Recrutamos 132 pescadores artesanais cadastrados nas respectivas colônias de pescadores Z-22 em Ipueiras-TO e Porto Nacional-TO. Destes, 77 pescadores participaram da pesquisa (Figura 1). Todos os participantes realizaram o exame parasitológico de fezes a caracterização demográfica está descrita na Tabela 1. Os aspectos socioeconômicos da amostra estão descritos na Tabela 2, e os aspectos sanitários na Tabela 3.

Figura 1. Participantes elegíveis para a pesquisa.

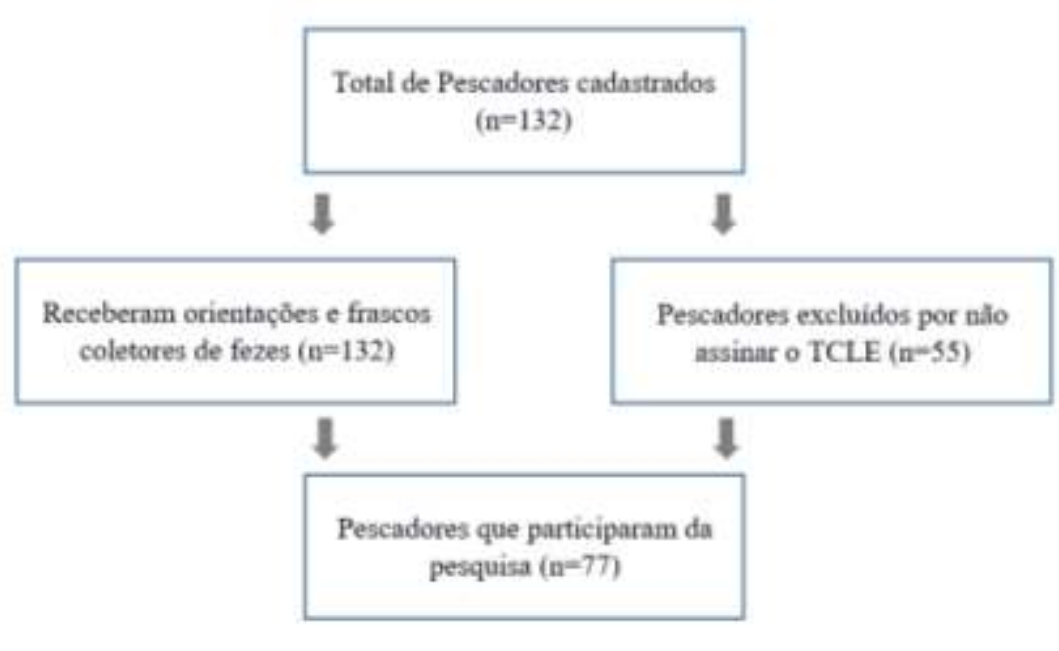

Fonte: Autores.

Em relação às amostras analisadas, $17,78 \%$ de Ipueiras e 28,13\% de Porto Nacional não receberam diagnostico por inconsistência do material analisado. No geral, prevaleceu amostras negativadas em Ipueiras 46,67\%, bem como em Porto Nacional 375\%. A prevalência e espécies de enteroparasitoses da população estudada estão descritas na Tabela 4.

Tabela 1. Associação entre aspectos demográficos e a presença de enteroparasitoses em pescadores no Norte do Brasil (n=77), no período de junho a setembro de 2019.

\begin{tabular}{|c|c|c|c|c|c|}
\hline \multirow{2}{*}{ Variáveis } & \multicolumn{2}{|c|}{ Ipueiras $(n=45)$} & \multicolumn{3}{|c|}{ Porto Nacional $(\mathrm{n}=32)$} \\
\hline & $\mathbf{n}$ & $\%$ & $\mathbf{n}$ & $\%$ & $\mathbf{p}^{*}$ \\
\hline \multicolumn{6}{|l|}{ Sexo } \\
\hline Masculino & 28 & 62,22 & 19 & 59,37 & \multirow{2}{*}{0,664} \\
\hline Feminino & 17 & 37,78 & 13 & 40,63 & \\
\hline \multicolumn{6}{|c|}{ Idade dos pescadores examinados } \\
\hline 18 a 27 anos & 3 & 6,67 & 1 & 3,13 & \multirow{5}{*}{0,803} \\
\hline 28 a 38 anos & 7 & 15,55 & 1 & 3,13 & \\
\hline 39 a 49 aos & 16 & 35,56 & 7 & 21,86 & \\
\hline 50 a 59 anos & 6 & 13,33 & 17 & 53,13 & \\
\hline 60 anos ou mais & 13 & 28,89 & 6 & 18,75 & \\
\hline
\end{tabular}

* Teste Qui-quadrado. Fonte: Autores. 
Tabela 2. Associação entre os aspectos socioeconômicos e a presença de enteroparasitoses em pescadores no Norte do Brasil ( $\mathrm{n}=77)$, no período de junho a setembro de 2019.

\begin{tabular}{|c|c|c|c|c|c|}
\hline \multirow[t]{2}{*}{ Variáveis } & \multicolumn{2}{|c|}{ Ipueiras $(n=45)$} & \multicolumn{2}{|c|}{ Porto Nacional $(n=32)$} & \multirow[t]{2}{*}{$\mathbf{p}^{*}$} \\
\hline & n & $\%$ & $\mathbf{n}$ & $\%$ & \\
\hline \multicolumn{6}{|l|}{ Escolaridade } \\
\hline Analfabeto/Fundamental I incompleto & 13 & 28,89 & 13 & 40,62 & \multirow[t]{5}{*}{0,996} \\
\hline Ensino fundamental I completo/Fundamental II incompleto & 16 & 35,56 & 16 & 50,00 & \\
\hline Fundamental II completo/Médio incompleto & 5 & 11,11 & 0 & 0,00 & \\
\hline Médio completo/superior incompleto & 8 & 17,77 & 3 & 9,38 & \\
\hline Superior completo & 3 & 6,67 & 0 & 0,00 & \\
\hline \multicolumn{6}{|l|}{ Renda } \\
\hline Menor ou igual a 1 salário mínimo & 21 & 46,67 & 20 & 62,50 & \multirow[t]{4}{*}{0,960} \\
\hline Entre 1 a 2 salários mínimos & 21 & 46,67 & 9 & 28,13 & \\
\hline Acima de 3 salários mínimos & 3 & 6,66 & 1 & 3,13 & \\
\hline Não respondeu & 0 & 0,00 & 2 & 6,24 & \\
\hline
\end{tabular}

* Teste Qui-quadrado. Fonte: Autores.

Tabela 3. Associação entre os aspectos sanitários e a presença de enteroparasitoses em pescadores no Norte do Brasil (n=77), no período de junho a setembro de 2019.

\begin{tabular}{|c|c|c|c|c|c|}
\hline \multirow[t]{2}{*}{ Variáveis } & \multicolumn{2}{|c|}{ Ipueiras $(n=45)$} & \multicolumn{2}{|c|}{ Porto Nacional $(n=32)$} & \multirow[t]{2}{*}{ p* } \\
\hline & $\mathbf{n}$ & $\%$ & $\mathbf{n}$ & $\%$ & \\
\hline \multicolumn{6}{|l|}{ Tipo de água de consumo } \\
\hline Rede de abastecimento local & 31 & 68,89 & 25 & 78,12 & 0,081 \\
\hline Poço & 14 & 31,11 & 7 & 21,88 & \\
\hline \multicolumn{6}{|l|}{ Condição da rua onde mora } \\
\hline Pavimentada / Asfaltada & 22 & 48,89 & 20 & 62,50 & 0,649 \\
\hline Terra / Cascalho & 23 & 51,11 & 12 & 37,50 & \\
\hline \multicolumn{6}{|l|}{ Banheiro } \\
\hline Sim & 44 & 97,78 & 32 & 100,00 & 0,926 \\
\hline Não & 1 & 2,22 & 0 & 0,00 & \\
\hline
\end{tabular}

* Teste Qui-quadrado. Fonte: Autores.

Tabela 4. Prevalência e Espécies de Enteroparasitoses em pescadores no Norte do Brasil (n=77), no período de junho a setembro de 2019.

\begin{tabular}{|c|c|c|c|c|}
\hline \multirow[t]{2}{*}{ Variáveis } & \multicolumn{2}{|c|}{ Ipueiras $(n=45)$} & \multicolumn{2}{|c|}{ Porto Nacional $(\mathrm{n}=32)$} \\
\hline & $\mathbf{n}$ & $\%$ & $\mathbf{n}$ & $\%$ \\
\hline Participante & 8 & 17,78 & 9 & 28,13 \\
\hline Não contribuiu com amostra & 14 & 31,11 & 11 & 34,38 \\
\hline Parasitados & 2 & 4,44 & 1 & 3,13 \\
\hline Poliparasitados & 21 & 46,67 & 12 & 37,50 \\
\hline \multicolumn{5}{|l|}{ Negativados } \\
\hline Espécies & \multicolumn{2}{|c|}{ Ipueiras $(n=16)$} & \multicolumn{2}{|c|}{ Porto Nacional $(n=11)$} \\
\hline Coli & 4 & 28,57 & 1 & 9,09 \\
\hline Nana + Coli & 0 & 0,00 & 1 & 9,09 \\
\hline Butschlii & 0 & 0,00 & 1 & 9,09 \\
\hline Nana & 8 & 57,14 & 6 & 54,54 \\
\hline Giardia Lamblia & 0 & 0,00 & 1 & 9,09 \\
\hline Coli + Lamblia & 2 & 14,29 & 0 & 0,00 \\
\hline
\end{tabular}

Fonte: Autores. 


\section{Discussão}

\section{Desfecho- Principais resultados}

Ocorreu uma baixa adesão do público ao projeto, a maioria são pescadores do sexo masculino, tem idade de 39 a 49 anos, a maior parte deles não terminou o ensino fundamental, mais da metade possuem renda per capita menor ou igual a um salário mínimo, quase a totalidade consome água proveniente da rede de abastecimento local e moram em ruas pavimentadas. Houve baixa prevalência de parasitos intestinais de interesse médico, não foram positivados a presença de helmintos nas análises das fezes dos pescadores.

\section{Interpretação}

Em relação à distribuição quanto ao sexo e idade, observamos uma distribuição parecida entre as duas colônias quanto ao gênero dos pescadores examinados. Seguindo a tendência de distribuição no estado do Tocantins que é de $67 \%$ masculinos e 33\% femininos (Sousa, 2013). Quanto a distribuição por idade dos participantes a maioria tinha idade de 39 a 49 anos em Ipueiras 35,56\% e 50 a 59 anos em Porto Nacional 53,13\%. Estudos em outras colônias de pescadores do estado demonstraram que $70 \%$ dos pescadores enquadram-se na faixa etária de 40 a 60 anos, corroborando para uma característica comum entre os pescadores do estado do Tocantins (Sousa et al., 2017; Sousa, 2013).

\section{População com baixa escolaridade}

Uma boa parte dos pescadores não concluíram o ensino fundamental 35,56\% dos pescadores de Ipueiras e 50\% dos pescadores de Porto Nacional. A baixa escolaridade e a ausência de promotores de saúde elevam o indivíduo à condição de vulnerabilidade para aquisição de doenças (Barbosa et al., 2009). Por outro lado, ações educativas direcionadas à prevenção de parasitoses representam uma boa estratégia de aprendizado, assim, pessoas educadas produzem mudanças práticas e habituais de proteção à saúde colaborando para diminuição do índice de adoecimento por doenças parasitárias.

\section{População com baixa renda}

A renda salarial dos pescadores é inferior à média salarial dos trabalhadores formais tanto em Porto Nacional como em Ipueiras que têm ambas médias de 2,2 salários mínimos por pessoa. A renda é fator determinante para a melhoria da qualidade de vida, nas populações vulneráveis o elevado índice de enteroparasitoses é reflexo imediato do baixo índice educacional e distribuição mínima de renda nessas comunidades (Ferraz et al., 2014)

\section{Procedência da água de consumo humano}

Em ambas colônias os pescadores utilizam com mais frequência água tratada do sistema de abastecimento local, moram em ruas asfaltadas e em suas residências e possuem banheiros em suas residências. A maior fonte de contágio por parasitos intestinais é mediante a ingestão de água contaminada, isso acontece principalmente em locais com pouca infraestrutura sanitária (Campos \& Chaves, 2016). Neste estudo os aspectos relacionados ao saneamento básico, como provimento da água e condições de moradia podem ter contribuído positivamente para os baixos índices de contaminação por parasitos intestinais patogênicos.

\section{Parasitos intestinais encontrados}

Nas duas comunidades os maiores índices de contaminação foram por Endolimax nana, que é um protozoário comensal. Toda via, nas duas colônias de pescadores observou-se o índice de contaminação superior a 35\% por parasitos 
intestinais, isto revela que a presença destes parasitos indica a existência de contaminação fecal oral podendo elevar a contaminação de parasitos intestinais patogênicos (Neves et al., 2005).

Os achados permitem estabelecer a relação dos poucos índices de morbidades ocasionadas por enteroparasitoses intestinas às boas condições de moradia e saneamento básico apresenta pela população estudada.

A maior parte das amostras analisadas foram negativadas em ambas as colônias de pescadores refutando a hipótese da existência de elevada prevalência de parasitoses intestinais em colônias de pescadores do estado do Tocantins de acordo com os achados neste inquérito parasitológico. Os achados que mostram o baixo índice educacional da população nos remetem ao entendimento da carência de ações educativas que combatam a contaminação por parasitos intestinais.

\section{Devolutiva dos resultados}

Os resultados obtidos foram devolvidos aos pescadores por meio da secretaria de saúde local pela enfermeira da comunidade e agentes comunitários sob supervisão médica.

\section{Limitações}

Como limitação deste estudo deve-se considerar que no Estado do Tocantins existem 35 comunidades pesqueiras catalogadas e registradas, o presente estudo fora realizado em apenas duas destas comunidades servindo assim de prognóstico do cenário atual, não caracterizando uma realidade fidedigna da situação em todo território tocantinense.

\section{Generalização}

Estudos da prevalência de infecções intestinais em pescadores são pouco explorados por decorrência de dificuldades de acesso, apontando para necessidade de novos estudos que complementem ou acrescentem informações sobre o tema. Todavia os achados nos direcionam para o entendimento que o combate das parasitoses intestinais deva ser baseado nos índices de prevalência e condições de vida dos pescadores.

\section{Conclusão}

Na região Norte do Brasil houve prevalência > 50\% de Endolimax Nana em pescadores parasitados.

\section{Agradecimentos}

Aos pescadores artesanais; e à Embrapa, Pesca e Aquicultura.

\section{Referências}

Andrade, E. C. De, Leite, I. C. G., Rodrigues, V. D. O., \& Cesca, M. G. (2010). Parasitoses intestinais: Uma revisão sobre seus aspectos sociais, epidemiológicos, clínicos e terapêuticos. Revista APS, 13(2), 231-240.

Barbosa, L. de A., Sampaio, A. L. A., Melo, A. L. A., Macedo, A. P. N. de, \& Machado, M. de F. A. S. (2009). A educação em saúde como instrumento na prevenção de parasitoses. Revista Brasileira Em Promoção Da Saúde, 22(4), 272-278. https://doi.org/10.5020/18061230.2009.p272

Brasil. (2019). Pesca no Brasil - Ministério da Agricultura, Pecuária e Abastecimento.

Campos, A. G., \& Chaves, J. V. (2016). Perfil laboral dos pescadores artesanais no brasil: insumos para o programa seguro defeso. política em foco, 60.

Cruz, D. T. da, Ribeiro, L. C., Vieira, M. de T., Teixeira, M. T. B., Bastos, R. R., \& Leite, I. C. G. (2011). Prevalência de quedas e fatores associados em idosos. Revista de Saúde Pública, 46(1), 138-146. https://doi.org/10.1590/s0034-89102011005000087

Dos Santos Carvalho, O., Guerra, H. L., Campos, Y. R., Caldeira, R. L., \& Massara, C. L. (2002). Prevalência de helmintos intestinais em três mesorregiões do Estado de Minas Gerais. Revista Da Sociedade Brasileira de Medicina Tropical, 35(6), 597-600. https://doi.org/10.1590/s0037-86822002000600009

Ferraz, R. R. N., Barnabé, A. S., Porcy, C., D’Eça Júnior, A., Feitosa, T., \& Figueiredo, P. de M. (2014). Parasitoses intestinais e baixos índices de Gini em 
Research, Society and Development, v. 10, n. 13, e450101321599, 2021

(CC BY 4.0) | ISSN 2525-3409 | DOI: http://dx.doi.org/10.33448/rsd-v10i13.21599

Macapá (AP) e Timon (MA), Brasil. Cadernos Saúde Coletiva, 22(2), 173-176. https://doi.org/10.1590/1414-462x201400020010

Freitas, M. B. de, \& Rodrigues, S. C. A. (2015). Determinantes sociais da saúde no processo de trabalho da pesca artesanal na Baía de Sepetiba , estado do Rio de Janeiro. Saúde Soc. São Paulo, 24(3), 753-764. https://doi.org/10.1590/S0104-12902015126063

IBGE. (2010a). IBGE | Cidades@ | Tocantins | Porto Nacional | História \& Fotos.

IBGE. (2010b). Tabela 211: Estabelecimentos de saúde.

Malta, M., Cardoso, L. O., Bastos, F. I., Magnanini, M. M. F., \& Silva, C. M. F. P. da. (2010). Iniciativa STROBE: subsídios para a comunicação de estudos observacionais. Revista de Saúde Pública, 44(3), 559-565. https://doi.org/10.1590/s0034-89102010000300021

Marques, A. P. (2003). Manual de Goniometria (2ed ed.). Manole. https://books.google.com/books?id=hV0iCgAAQBAJ\&pgis=1

Marzagão, M., Rezende, W., Ii, D. A., Cléa, M., Ii, B., Gonçalves, H., Oliveira, N., \& Iii, B. (2010). Ocorrência de parasitoses intestinais em habitantes do município de Pará de Minas, MG - Brasil Occurrence of intestinal parasitosis among inhabitants of Pará de Minas , MG - Brazil. Rev. Bras. Farm., 91(4): 183-8, 2010, 91(4), 183-188.

Meirelles, B. H. S., Arruda, C., Simon, E., Vieira, F. M. A., Cortezi, M. D. V., \& Natividade, M. S. L. (2010). Condições associadas à qualidade de vida dos idosos com doença crônica TT - Conditions associated with the quality of life of elderly with chronic disease. Cogitare Enferm, 15(3), 433-440.

Neves, D. P., Wagner, R., Vitor, D. A., Mastigophora, S., Siqueira, A. M., Suzan, M., Michalick, M., Americana, L. T., Genaro, O., Reis, A. B., \& Genaro, O. (2005). Parasitologia Humana - Neves $11^{\mathrm{a} e d}$. Parasitologia Humana, 127-138. https://doi.org/10.1086/521246

Santos, P. H. S., Barros, R. de C. S., Gomes, K. V. G., Nery, A. A., \& Casotti, C. A. (2017). Prevalence of intestinal parasitosis and associated factors among the elderly. Revista Brasileira de Geriatria e Gerontologia, 20(2), 244-253. https://doi.org/10.1590/1981-22562017020.160137

Sousa, D., Kato, H., \& Milagres, C. (2017). Perfil socioeconômico e tecnológico dos pescadores de Xambioá, Estado de Tocantins. 5, 113-121. https://doi.org/10.2312/ActaFish.2017.5.3.113-121

Sousa, D. N. de. (2013). Documentos 2 Diagnóstico preliminar da extensão pesqueira no estado do Tocantins. EMBRAPA Pesca e Agricultura, 1, 50. 